\title{
Introduction: From a Philosophical Point of View
}

\author{
Rasmus Grønfeldt Winther
}

Received: 12 February 2009/Accepted: 12 February 2009/Published online: 4 March 2009

(C) Springer Science+Business Media B.V. 2009

The six articles of this section of the special issue explore philosophical questions of comparative biology, very broadly construed. Under this category, I place systematics, classification, and historical Darwinian evolutionary theory. Classic epistemic and metaphysical questions of comparative biology include: Are species natural kinds or individuals? What are the proper units of the biological hierarchy(ies) pertinent to evolution and classification? What is the fundamental relation between the Tree of Life and the Linnean Hierarchy? Why was Darwin's (putative?) focus on variational rather than typological thinking so revolutionary? How does phylogenetic inference and reasoning work? Moreover, how should it work? How are parts of complex historical systems individuated? What would a historically sensitive notion of the biological function of structures, behaviors and characters look like? These questions have been investigated from a philosophical point of view at least since the seminal work of philosophers who started analytical philosophy of biology during the 1960s and 1970s, including David Hull, Michael Ruse, Elliot Sober, and William Wimsatt. Biologists such as Richard Lewontin, Ernst Mayr, and Stephen J. Gould have also contributed significantly to setting the question agenda on these matters.

We find ourselves today at a dizzying time in biological research. Astounding data-driven progress is being made in a variety of broad biological fields including: (1) genomics, proteomics, and systems biology, (2) biodiversity and ecology, and (3) evolutionary developmental biology ("evo-devo"). This is not the place to substantiate the reality and depth of these research fields; I trust that that is clear and present to the reader. However, this is a place to argue that each of these growth fields relies heavily - if not explicitly, then certainly implicitly—on comparative biology. Briefly consider the following questions for each area: How do we investigate and discover the function of a particular gene or molecule? We perform a comparative analysis of that gene sequence or molecular structure across a range

R. G. Winther $(\square)$

Philosophy Department, University of California, 1156 High St., Santa Cruz, CA 95064, USA

e-mail:rgw@ucsc.edu; rgwinther@gmail.com 
of related taxa. How do we identify and individuate the species of particular ecosystems whose distribution patterns and numbers we would like to map? We appeal to the species inventory given to us by taxonomists. How do we justify new or revised homology claims in evolutionary developmental biology? Again, knowledge and methods provided by comparative biology are crucial.

We see that comparative biology is essential for many of the fastest-growing and revolutionary biological fields of our day. Thus, it would behoove us to philosophically explore and critically discuss the practice and theory, the epistemology and metaphysics, and the facts and values, of comparative biology. If philosophers desire to help state-of-the-art biological research be more efficient, critical, and honest, an excellent place to start would be with analyzing comparative biology. Indeed, this can be seen as the first main goal of "From a Philosophical Point of View".

For the remainder of this introduction, I would like to explore three novel issues relevant to comparative biology that emerge from the articles of this section, "From a Philosophical Point of View":

\section{1. futurism (Griffiths and Rieppel)}

2. Epistemological utility of classification (Brigandt and Love)

3. Scientific values and objectivity (McManus and Winther)

These issues have deep roots in the questions mentioned in the first paragraph above. Yet they are also new in part because they are responding to changes in contemporary biological research. This shows that neither philosophy nor its object of study (comparative biology, in this case) is impervious to ongoing progress in biological research. A second main goal of this section, then, is to investigate conceptual, methodological, and empirical changes in comparative biology that are caused by the research fields for which it is practically a necessary, transcendental condition of possibility.

To summarize: comparative biology is essential to biological research in genomics, ecology, and evo-devo and hence worth exploring philosophically (Goal 1). Yet its methods and knowledge are also influenced by biological research; philosophical analysis can and should therefore also provide insight into the complex theoretical and practice relations across various porous disciplinary boundaries in the biological sciences (Goal 2). Let us turn to the three issues.

\section{Futurism}

Comparative biology is concerned with the past. It reconstructs the history of life, traces genealogies of organism parts, and describes historical constraints on the operation of natural selection. But Griffiths' and Rieppel's articles in this section make an interesting, complementary, suggestion: in a number of important respects, comparative biology is also about the future. I will call this family of views futurism (Rieppel uses "futuralism").

First, Griffiths ("In What Sense Does 'Nothing Make Sense Except in the Light of Evolution'?") suggests that a forward-looking perspective on survival and reproduction is necessary for correctly individuating both the higher-level relevant 
capacities of a system (organism) as well as the more basic parts and processes that causally contribute to those relevant capacities. Griffiths cogently argues that (1) parts and processes are not primitively available as basic biological furniture- they must be identified and abstracted in some manner and (2) a Millikan proper-function account appealing to past history must itself be forward-looking about the survival and reproduction of those very parts and processes in the past. For these two reasons, Griffiths feels that the individuation of parts and processes must rely on a forward-looking, evolutionary (and ecological) perspective. Since Griffiths is also interested in the problem of homology, it is important to note that he holds that there is a productive theoretical interplay between the individuation of parts and processes through homology, on the one hand, and through a forward-looking selective analysis, on the other hand. A further philosophical analysis of exactly how this interplay could be achieved would be of interest.

Second, Rieppel ("Species as a Process") develops a new account of species, which defines them with respect to their future potential as "open-ended bundles of processes" rather than in terms of "origin essentialism". Rieppel's article is a contribution to the metaphysics of species. While Rieppel does accept the historicity of species, he moves beyond the Ghiselin-Hull Species as Individuals (SAI) thesis in two ways: (1) species are also natural kinds, in the sense of homeostatic property cluster natural kinds á là Richard Boyd and (2) species - as well as the homeostatic mechanisms that ensure their integration as a natural biological units-can be disintegrated, (re)shuffled and (re)created through a broad variety of ecological, developmental and historical mechanisms that operate at various spatial, temporal, and material scales and levels. The second point is futurism. As a whole, Rieppel's article attempts to synthesize and overcome (i.e., aufhebung) a series of delicate, complex and productive dichotomies: (1) past and future, (2) essentialism and dynamism, (3) sameness and change, and (4) individuals and kinds. His implicit call to develop a theoretical biology that emphasizes Systems and Process is promising.

\section{Epistemological Utility of Classification}

Comparative biology is interested in classifying the buzzing, blooming confusion of biological units in the world, especially species and homologues. A common assumption of this classificatory theme is that diverse species and homologues exist in the actual world and that our efforts should therefore aim at finding the single best, objective classification. Love's and Brigandt's articles challenge this assumption in an interesting way. While they share the view that classifications either qua typologies (Love) or qua natural kinds (Brigandt) are an integral aspect of comparative biology, they believe that philosophical effort should be invested in epistemological rather than metaphysical matters. That is, rather than continue asking what species or homologues really are and whether our classifications can ever be objective, realistic, and counterfactually supporting (i.e., metaphysical issues), they suggest shifting our philosophical attention to investigations of actual classificatory practices and ask to what range of purposes, aims, and functions we can put our classificatory reasoning patterns (i.e., epistemological issues). 
What then happens to the core metaphysics of species and homologues? Here their analyses diverge in a productive manner. Love brackets metaphysical questions altogether-he adopts a sort of deflationism or, better yet, silentism about metaphysics. In contrast, Brigandt insists on reality as complex; different epistemological strategies (i.e., species as individuals vs. species as homeostatic property cluster kinds) capture different orthogonal aspects of this complex reality. Either way, their philosophical commitments are with epistemology, reasoning, and practice rather than with metaphysics, reference, and reality. Let us explore each article in turn.

First, Love ("Typology Reconfigured: From the Metaphysics of Essentialism to the Epistemology of Representation") analyzes typological thinking. He seeks to rescue it from the typical demonization to which it has been subject ever since its official baptism by Ernst Mayr. How does he save it? Rather than attempt to provide ever-more sophisticated scholastic arguments justifying the metaphysical reality of types for just a few disciplines of the life sciences (especially comparative biology), Love finds it to be a more useful strategy to point to the widespread use of typologies across the biological sciences. Love suggests that they are present in molecular and structural biology, evolutionary genetics, functional morphology, and systematics. In addition to motivating their broad extension, Love also offers a philosophical analysis of typologies: they are epistemic reasoning tools that allow us to classify, represent, and explain through abstraction, idealization, approximation, and generalization. In short, Love's article outlines a useful epistemological research program focused on the nature and power of epistemic typologies.

Second, Brigandt ("Natural Kinds in Evolution and Systematics: Metaphysical and Epistemological Considerations") calls for a renewed role for natural kinds in evolution and systematics. Brigandt wishes to stress the epistemic role of natural kinds in investigations surrounding species and homologues. For instance, rather than seeing the SAI and the "essentialist" Species as Natural Kinds (SANK) thesis as mutually exclusive, Brigandt suggests that they are complementary. Since each addresses different questions and expresses different assumptions, they are useful in different contexts. For example, the SAI thesis provides much more traction in evolutionary historical studies of species (e.g., in processual studies of speciation) while the SANK thesis grants more understanding for strictly taxonomic studies, especially alpha and beta taxonomy. Brigandt's epistemic pluralism does not preclude a realist position: reality is just complex! In order to develop his complementarity thesis, Brigandt must present a biologically plausible position of natural kinds. This he does through a direct and novel articulation of the epistemic utility of the homeostatic property cluster kind concept. This article presents a strong defense of an epistemic reorientation of the debate(s) on biological natural kinds, species, and homologues.

\section{Scientific Values and Objectivity}

Comparative biology, as so many other sciences, often misunderstands and downplays the role of modeling assumptions and biases, background theories, and 
epistemic and social values in its own knowledge production. The proclaimed aim is to eliminate these "subjectivist" and all-too-human aspects of science. After all, these elements only infect and distort the epistemic processes and theoretical products of science. Objective knowledge requires objective inquiry. McManus' and Winther's articles take issue with this interpretation of the role of values, assumptions, and biases in cladistic analysis and character analysis. They argue that these are irreducible and inevitable aspects of comparative biology. Rather than eliminate values, assumptions, and biases, we should celebrate and work with them in the best way possible-i.e., have agreement and objectivity as a regulative ideal.

Two important consequences follow when we take seriously the inevitable role of values, assumptions, and biases. First, we must admit the possibilities of (1) rational disagreements resulting in an impasse and (2) the potential failure of objectivity. Failures of agreement and objectivity are indeed often the case in science, at least for some periods of time. Second, it becomes extremely difficult to be a naïve or strong realist. The rich epistemic and social machinery, as well as the diversity of such machineries, are now the object of philosophical study. Even if we remain committed to the existence of a reality (as both McManus and Winther do), through the philosophical exploration of the limitations, weaknesses, and disagreements of the knowledge production machinery, it becomes clear that reality is difficult to know and is always mediated. Moreover, a philosophical investigation of comparative biology also shows that reality can legitimately be rendered in multiple ways and that some of those renditions may be orthogonal or even contradictory. These two consequences need not be interpreted as negative. Rather, they give a more complete picture of knowledge production, and potential failure thereof, in comparative biology. Perhaps with this picture in hand, recipes for future agreement and objectivity can be articulated. Let us explore each article in turn.

First, McManus ("Rational Disagreements in Phylogenetics") argues that a rational disagreement exists between two schools of phylogenetic analysis: maximum parsimony (MP) and maximum likelihood (ML). Following Kuhn, he holds that scientific theories contain a set of values and epistemic norms (virtues) in addition to symbolic generalizations (i.e., laws), ontological assumptions, and exemplars. The disagreement between MP and ML can be traced to the adoption of differing epistemic norms. And since there is no meta-theoretical objective "view from nowhere," we cannot-and should not-choose rationally between these schools of theories and practices. McManus holds that these traditions are currently at an impasse and that it remains unclear what the next stop would be. In order to explicate the values, assumptions, and biases of these schools, McManus divides their respective "Style of Modeling" into four stages. This instructive staging heuristic provides a way of understanding the modeling process of these two phylogenetic schools.

Second, Winther ("Character Analysis in Cladistics: Abstraction, Reification, and the Search for Objectivity") explores character analysis in cladistic methodology. I argue that there are certain criteria that a character must meet if it is to be objective (i.e., be a primary homology). Each objectivity criterion involves particular norms and practices. Now, the abstraction process of character identification, individuation, and measurement is always theory-laden-both 
robustly real as well as uncritically reified characters can therefore be inferred using theory-laden abstraction. The key for detecting robustly real, objective characters is for abstraction to comply with the objectivity criteria. Ultimately, the problem of character analysis is important because erroneous, reified characters will still give us a cladogram, albeit an inaccurate one, with grossly mistaken "homologies". It is also important to note, that even an objective character may turn out not to be phylogenetically homologous; this is because even good characters can conflict with one another when constructing phylogenies. Two of my objectivity criteria are not usually discussed in comparative biology: causal grounding and inter-disciplinary communication. However, the philosophical literature shows how both criteria provide useful norms and practices.

The issues of futurism, epistemological utility of classification, and scientific values and objectivity thus allow us to ask new sorts of questions about comparative biology and its crucial role in ongoing biological research. Moreover, the great care that each of these articles takes with the subtle details of biological research also sheds light on the multiple ways that genomics, ecology, and evolutionary developmental biology are influencing comparative biology. Comparative biology will remain crucial to the life sciences. As philosophers, we would do well to work towards understanding it, particularly in these exciting and worrisome times of "Darwin Year," at the beginning of the twenty-first century. 